\title{
Coherent Diffractive Imaging Using Phase Front Modifications
}

\author{
I. Johnson, ${ }^{1}$ K. Jefimovs, ${ }^{1, *}$ O. Bunk, ${ }^{1}$ C. David, ${ }^{1}$ M. Dierolf, ${ }^{1}$ J. Gray,${ }^{2}$ D. Renker, ${ }^{1}$ and F. Pfeiffer ${ }^{1,3}$ \\ ${ }^{1}$ Paul Scherrer Institut, 5232 Villigen PSI, Switzerland \\ ${ }^{2}$ Lawrence Livermore National Laboratory, Livermore, California 94550, USA \\ ${ }^{3}$ Ecole Polytechnique Fédérale de Lausanne, 1015 Lausanne, Switzerland
}

(Received 17 January 2008; published 17 April 2008)

\begin{abstract}
We introduce a coherent diffractive imaging technique that utilizes multiple exposures with modifications to the phase profile of the transmitted wave front to compensate for the missing phase information. This is a single spot technique sensitive to both the transmission and phase shift through the sample. Along with the details of the method, we present results from the first proof of principle experiment. The experiment was performed with $6.0 \mathrm{keV}$ x rays, in which an estimated spatial resolution of $200 \mathrm{~nm}$ was achieved.
\end{abstract}

DOI: 10.1103/PhysRevLett.100.155503

The high degree of coherence of today's x-ray sources has promoted the development of various diffractive imaging techniques that are not hindered by imperfections in optical elements. In general, coherent diffractive imaging (CDI) uses the intensity distribution of a diffracted wave to deduce information about the specimen, such as attenuation and phase shift distributions. A common challenge in CDI is recovering the phase component of the wave field, which is lost in the measurement. Iterative algorithms are commonly used to retrieve the phase information [1-4]. Since the first demonstration by J. Miao et al. [5], CDI has proven successful in many instances [6-9]. These techniques attempt to reconstruct the image of a specimen from a single diffraction pattern. This procedure requires a substantial number of iterations, has difficulties with complexvalued exit waves, and may not lead to a unique solution. Furthermore, one of the most stringent limitations to these methods is the need for isolated objects (the so-called support constraint).

These limitations may be overcome with complementary information from multiple diffraction patterns. The Ptychographical Iterative Engine (PIE) [10-12] has recently demonstrated the power of combining multiple diffraction data sets to ease the retrieval of the phase information. In this technique, overlapping illuminations lead to the complementary information. Here, we report on a new technique that takes advantage of phase front modifications $[13,14]$ after the sample to generate different diffraction patterns. As a result, the altered exit waves force each exposure to have a unique interference pattern and compensate for the lack of phase information. Similar to the PIE method, complementary information directs the convergence of a numerical algorithm and solves ambiguities in the reconstruction. In contrast to the conventional techniques, a sharp support is not needed and extended specimens may be imaged. Additionally, since both the computational and data acquisition times can be short (seconds), this new technique provides the opportunity for realtime image reconstruction. With regards to microscopy, this technique is optimal for specimens that fit within
PACS numbers: 61.05.cp, 42.30.Rx, 42.30.Wb

a coherent area of the radiation, in our case a few micrometers in size. In this Letter, we report on the technique and results from the first demonstration experiment.

Coherent radiation is locally attenuated and phase shifted as it traverses the sample. In the description of this new technique, we will focus on the wave front in three distinct locations: the wave front incident on the sample $\psi^{\text {inc }}$, the wave front just after the sample $\psi^{s}$, and the exiting wave front after the phase plate $\psi^{\text {exit }}$. The phase plate, $P$ translated by $\mathbf{r}_{i}$, is introduced just behind the sample to further modify the phase profile,

$$
\psi_{i}^{\text {exit }}(\mathbf{r})=\psi^{s}(\mathbf{r}) P\left(\mathbf{r}-\mathbf{r}_{i}\right) .
$$

Thus, the resulting far-field interference pattern is also altered. Independent diffraction patterns of the same illumination area on the sample ( $\psi^{s}$ is identical for all exposures) are recorded by shifting the phase plate with respect to the wave front after the sample. These independent exposures lead to an over-determination of the attenuation and phase shift through the sample.

The reconstruction algorithm cycles through these exposures. Starting with either an initial guess or the previous estimate of the wave front following the sample, $\psi^{s}$, the reconstruction algorithm calculates the exit wave front for the $i$ th illumination, Eq. (1). This exit wave front is then propagated to the far-field with a Fourier transformation. In the far-field, the phase information of the estimated complex interference pattern is untouched, while the amplitude distribution is replaced by the measured diffraction pattern. Back propagating this data corrected interference pattern with an inverse Fourier transformation generates a new estimate of the exit wave, $\psi_{i}^{\text {exit' }}$. A weighted combination of this new estimate and the previous estimate of the wave front following the sample produces an improved estimate of the wave front just after the sample,

$$
\psi^{s^{\prime}}(\mathbf{r})=[1-F(\mathbf{r})] \psi^{s}(\mathbf{r})+F(\mathbf{r}) \frac{P^{*}\left(\mathbf{r}-\mathbf{r}_{i}\right)}{\left|P\left(\mathbf{r}-\mathbf{r}_{i}\right)\right|^{2}} \psi_{i}^{\text {exit }}(\mathbf{r}) .
$$


This brings us to the next phase plate position or iteration, where $P^{*}$ is the complex conjugate of $P$, and $F$ is the relative update factor. The relative update factor biases the update in regions where the illumination on the sample has a higher intensity. In fact, it is directly proportional to the relative intensity of the illumination function across the sample,

$$
F(\mathbf{r})=\frac{\left|\psi^{\text {inc }}(\mathbf{r}) P\left(\mathbf{r}-\mathbf{r}_{i}\right)\right|}{\max \left[\left|\psi^{\text {inc }}(\mathbf{r}) P\left(\mathbf{r}-\mathbf{r}_{i}\right)\right|\right]} .
$$

After typically 10 to 20 iterations, the algorithm converges to a unique solution for $\psi^{s}$ in the plane of the phaseshifting aperture. It is the untouched wave front preceding the phase-shifting aperture and the complementary information in the diffraction plane from the various exposures that directs the reconstruction to a solution in the plane of the phase-shifting aperture. As in all phase retrieval methods, the sample illuminating wave front, $\psi^{\text {inc }}$, must be known in order to deduce the properties of the sample. Lastly, the attenuation and phase-shifting properties of the sample, $S$, are deduced by dividing out the illuminating wave front,

$$
S(\mathbf{r})=\frac{\psi^{s}(\mathbf{r})}{\psi^{\text {inc }}(\mathbf{r})}
$$

This technique is applicable to many types of coherent transmission microscopy: like laser light, electrons, and $\mathrm{x}$ rays. We have performed a demonstration experiment with $x$ rays at the microXAS beamline of the Swiss Light Source, Paul Scherrer Institut (PSI). A schematic of the experiment is shown in Fig. 1. An optimal x-ray energy of $6.0 \mathrm{keV}(\lambda \approx 2.1 \AA)$ was chosen to balance the transmission power of the $\mathrm{x}$ rays and the coherence length of the beam. A $10 \mu \mathrm{m}$ pinhole [15] 35 meters from the source selected a coherent portion $[16,17]$ of the beam coming from the double-crystal monochromator. This coherent radiation illuminated the test sample, a nanofabricated $16 \mu \mathrm{m}$ PSI logo, located $0.5 \mathrm{~mm}$ downstream from the pinhole. Half a millimeter further downstream came the transparent phase-modifying plate.

The ideal phase plate would populate the diffraction intensity uniformly in reciprocal space and, thus, interferes with all length scales of the specimen. The chosen circularly symmetric concentric ring structure (shown in Fig. 1) accomplishes this fairly well and is not complicated to fabricate with high precision. This structure was nanofabricated by electroplating gold through a polyimide mould. Details of the fabrication process may be found in Ref. [18]. It is composed of four concentric phase-shifting rings constructed from $680 \mathrm{~nm}$ of gold on a $3 \mu \mathrm{m}$ thick silicon membrane. At $6 \mathrm{keV}$, the rings result in about $\pi / 2$ phase shift and 44\% attenuation in the wave front [19].

In the far field, $3.2 \mathrm{~m}$ down stream, diffraction patterns were captured with a fiber coupled CCD detector (Photonic Science Hystar, effective pixel size $4.5 \mu \mathrm{m}$ ). Diffraction patterns of the sample were recorded with the phasemodifying plate in seven different lateral positions [20] with respect to the coherent illumination area on the sample. Thus, the final interference patterns are independent and carry identical information about the exit wave of the sample.

At each position, a short $(700 \mathrm{~ms})$ exposure was acquired to capture the intense central part of the diffraction pattern within the dynamic range of the detector. Long (7 s) exposures were also recorded at each position to obtain statistically significant data at higher spatial frequencies. These two images were combined into a high-dynamic range diffraction pattern. Of the $2048 \times 2096$ pixels of the camera, data from the central $1536 \times 1536$ pixels $\left(6.91 \times 6.91 \mathrm{~mm}^{2}\right)$ were selected. This corresponds to a maximum spatial frequency of about $3.3 \times 10^{-2} \mathrm{~nm}^{-1}$.

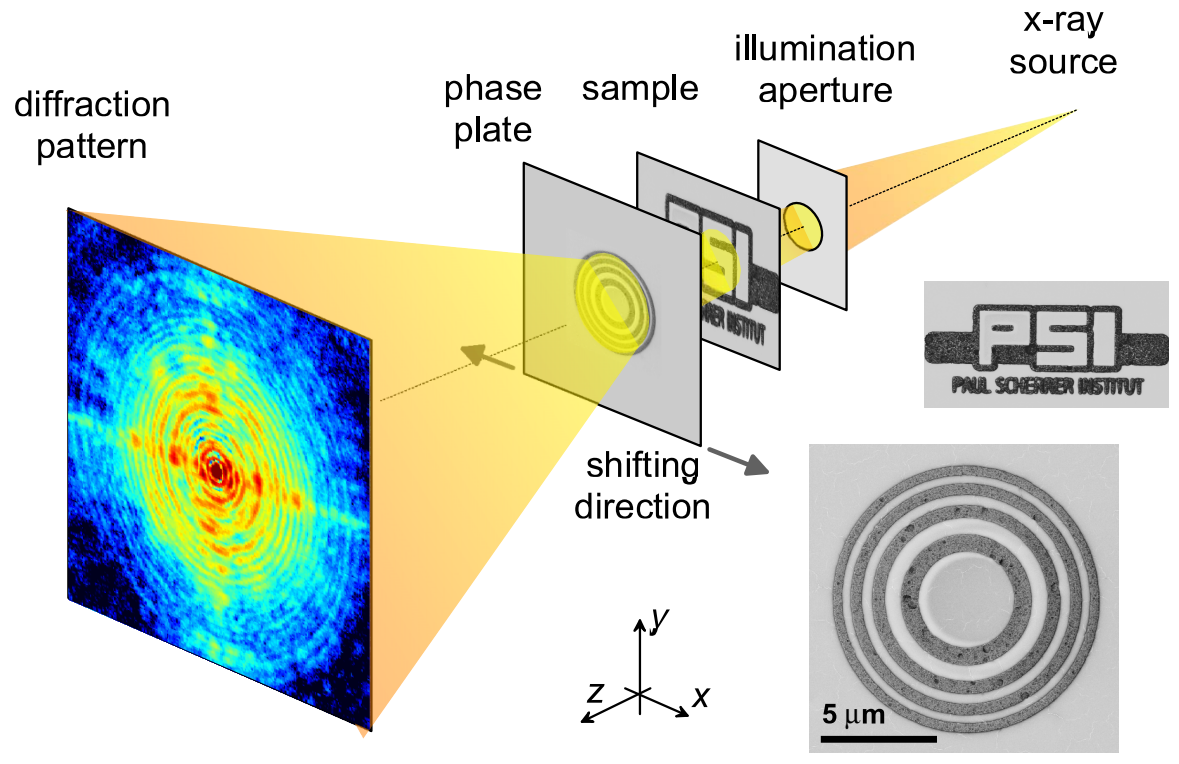

FIG. 1 (color online). Schematic of the experimental setup. 

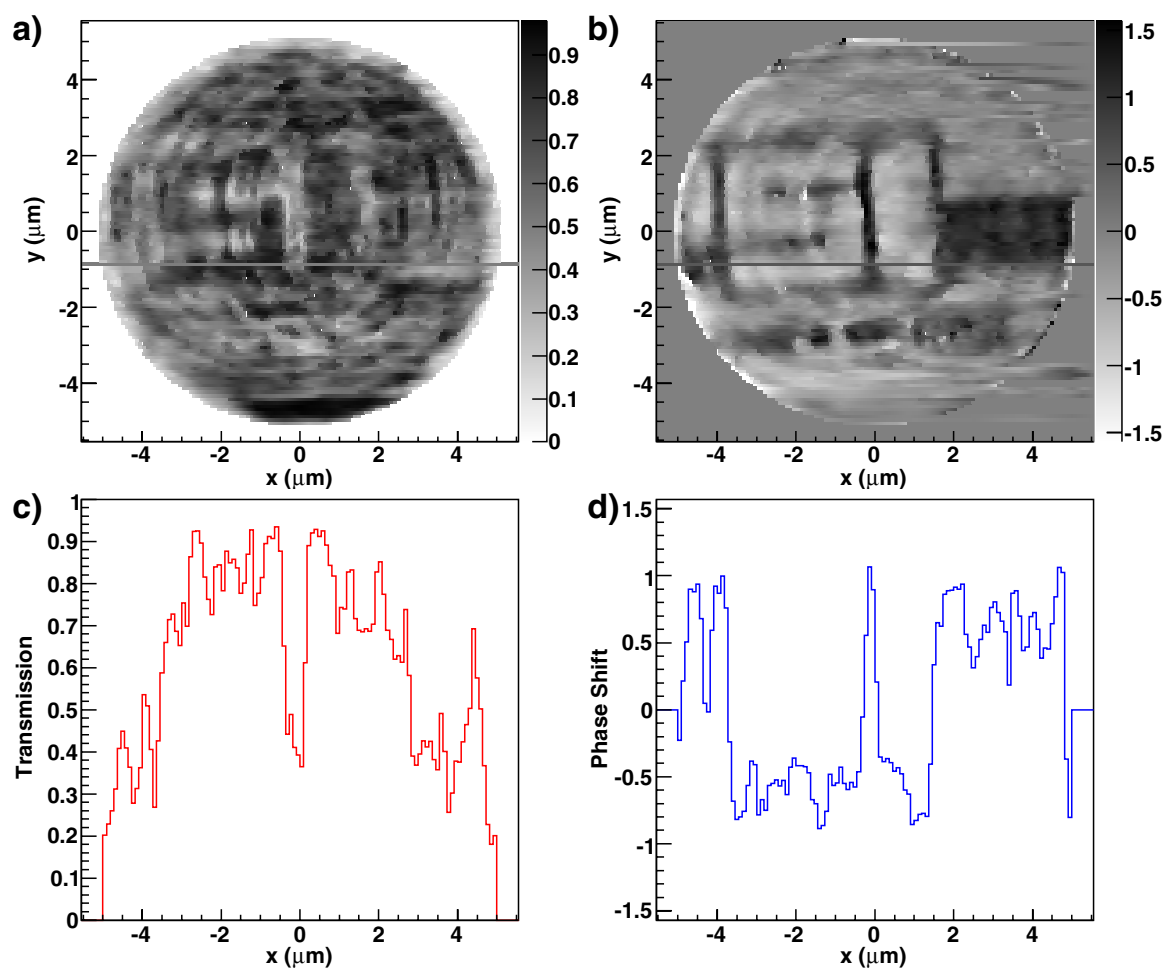

FIG. 2 (color online). Reconstructed transmission (a) and phase shift (b) images through the sample. The horizontal lines indicate the location of the profile transmission plot (c) and phase shift plot (d).

The short and long exposure images were also used to estimate the nonlinear response of the detector versus integrated flux, the illumination function and diffuse scattering background.

A constant attenuation $(a=0.5)$ and zero phase shift $(\theta=0)$ across the sample was assumed for the initial guess of the wave front following the sample, $\psi^{s}$. This guess provided the starting point for the reconstruction. The amplitude of the diffraction pattern from the exit wave $\psi_{i}^{\text {exit }}$ - the wave front following the phase-modifying plate, deduced from the product of the guess and the phasemodifying plate at the first position [Eq. (1)] — was replaced by the corresponding measured diffraction pattern data. Upon Fourier back transformation, the estimate of the wave front following the sample, $\psi^{s}$, was updated according to Eq. (2). Following further iterations, the algorithm quickly converged to the correct solution. The resulting reconstructed transmission and phase shift through the sample after 20 iterations of cycling through the 7 positions are shown in Fig. 2.

As the iteration number in the reconstruction increases, the change in the image becomes smaller and the image converges on the solution. The difference between images of adjacent iterations given by the invariant error metrics introduced by J. R. Fienup, Eq. 2 of [21], is shown in Fig. 3. The lower data points in the figure represent the convergence for the uniform starting guess described in the text above, while the higher points represent the average value and its standard deviation for 100 random starting guesses. The random starting guesses were both random in their attenuation and phase shift. It can be seen that regardless of the starting point, the multiple exposures quickly guide, within tens of iterations, the reconstruction to the $1 \%$ update level (level of Fig. 2). All initial guesses are di-

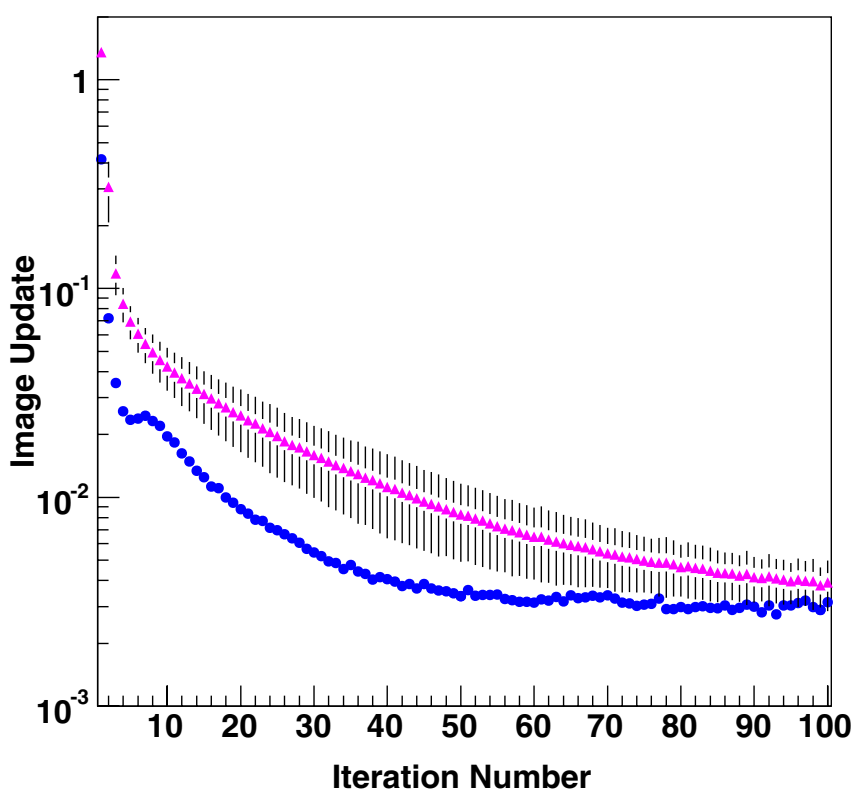

FIG. 3 (color online). Object update vs iteration number. The lower circles are the change in the image update for the uniform attenuation $(a=0.5)$ and zero phase shift starting guess; the upper triangles correspond to the average update progression for 100 random initial guesses. 
rected to the correct solution; furthermore, the speed of convergence is faster for the more realistic, uniform starting guess. This justifies our starting guess of a constant attenuation and phase shift across the sample.

In the $10 \mu \mathrm{m}$ diameter field of view of the reconstructed image [Fig. 2(a) and 2(b)] are the micrometer size letters "SI," the trailing strike, and the lower hundred-nanometer size letters of the PSI Logo (Fig. 1). The large "SI" letters are clearly readable in both the transmission and phase images. The boundaries of the letters are $680 \mathrm{~nm}$ high gold structures. They theoretically correspond to a $44 \%$ attenuation and a phase shift of 1.58 radians [opposite direction in the gray scales of Figs. 2(a) and 2(b)] relative to the surrounding $700 \mathrm{~nm}$ polyimide mould. The reconstructed transmission and phase shift in the gold regions are in good agreement with these expected values. This agreement is better seen in Figs. 2(c) and 2(d) where projections of the reconstructed images along the lines shown in Figs. 2(a) and 2(b) are plotted. A $400 \mathrm{~nm}$ wide gold bar separates the large " $S$ " and " $I$ " letters of the logo. In this region, the transmission drops from approximately $90 \%$ to $40 \%$, corresponding to $44 \%$ attenuation, the dip in Fig. 2(c) located at $-0.12 \mu \mathrm{m}$. The phase is also shifted from -0.65 to 0.95 radians, a 1.6 rad phase shift, as shown in the same location in Fig. 2(d). The overall 10\% attenuation in the transmission image is attributed to the $3 \mu \mathrm{m}$ silicon membrane that supports the sample.

In principle, the resolution of the technique is limited by the angular extent of statistically significant data. In this demonstration experiment, the resolution of both the transmission and phase shift is on the order of the limit posed by the pixel size of the reconstruction $95 \mathrm{~nm}$, which is fixed by the extent of the measured diffraction pattern. The transition - the lateral width between a change from $10 \%$ to $90 \%$ of the signal - of the sharp boundaries of the logo occur in less than a few pixel widths. From this, we estimate the resolution in both the transmission and phase shift images to be about $200 \mathrm{~nm}$. The imperfect contrast and noise in the reconstructed images are attributed to the experimental data. These artifacts are not present in noisefree computer simulations [22], thus confirming that the artifacts are not produced by the method.

In this Letter, we have presented a new technique that utilizes multiple exposures with modifications to the phase of the exiting wave front to direct the iterative reconstruction to the correct solution. It is similar to the common microscope where a single spot is placed into a focus; then the sample may be appropriately repositioned for further investigation. The technique overcomes the loss of the phase information, which leads to reconstruction ambiguities in other techniques. It is not limited to isolated specimens, and complex objects may be imaged in their natural environment. Furthermore, the independent, however complementary, diffraction patterns direct the iterative recon- struction to the correct solution in the well-defined plane of the phase plate.

The method is applicable and has a future in laser light, electron, and x-ray microscopy. In this Letter, the feasibility of the method has been demonstrated with the first proof of principle experiment, conducted with $\mathrm{x}$ rays. The fast convergence and data acquisition times make this technique suitable for both real time imaging and three-dimensional nanoscale tomography. These points lead us to believe that this technique with improved data quality can assist the investigation of small material science and biological samples.

We gratefully acknowledge the assistance of C. Borca, $\mathrm{X}$. Donath, D. Grolimund, and B. Meyer during the experiments. We also thank A. Menzel, D. K. Satapathy, and P. Thibault for fruitful discussions. This work has been performed at the Swiss Light Source, Paul Scherrer Institut, Villigen, Switzerland.

*Current affiliation: EMPA, 8600 Dübendorf, Switzerland

[1] R. W. Gerchberg and W. O. Saxton, Optik (Jena) 35, 237 (1972).

[2] J. R. Fienup, Appl. Opt. 21, 2758 (1982).

[3] V. Elser, J. Opt. Soc. Am. A 20, 40 (2003).

[4] S. Marchesini et al., Phys. Rev. B 68, 140101 (2003).

[5] J. Miao et al., Nature (London) 400, 342 (1999).

[6] G. J. Williams et al., Phys. Rev. Lett. 90, 175501 (2003).

[7] J. Miao et al., Proc. Natl. Acad. Sci. U.S.A. 100, 110 (2003).

[8] H. N. Chapman et al., Nature Phys. 2, 839 (2006).

[9] D. Shapiro et al., Proc. Natl. Acad. Sci. U.S.A. 102, 15343 (2005).

[10] H. M. L. Faulkner and J. M. Rodenburg, Phys. Rev. Lett. 93, 023903 (2004).

[11] J. Rodenburg et al., Phys. Rev. Lett. 98, 034801 (2007).

[12] O. Bunk et al., Ultramicroscopy 108, 481 (2008).

[13] K. A. Nugent et al., Phys. Rev. Lett. 91, 203902 (2003).

[14] F. Zhang et al., Phys. Rev. A 75, 043805 (2007).

[15] The aperture is composed of $\mathrm{Pt} / \mathrm{Ir}$ and was purchased from Plano GmbH.

[16] Vertical and horizontal coherence lengths: $\xi_{v} \approx 367 \mu \mathrm{m}$ and $\xi_{h} \approx 36 \mu \mathrm{m}$ (FWHM).

[17] F. Pfeiffer et al., Phys. Rev. Lett. 94, 164801 (2005).

[18] K. Jefimovs et al., Microelectron. Eng. 84, 1467 (2007).

[19] B.L. Henke, E. M. Gullikson, and J.C. Davis, X-ray Interactions: Photoabsorption, Scattering, Transmission, and Reflection at $E=50-30000 \mathrm{eV}, Z=1-92$, Atomic Data and Nuclear Data Tables Vol. 54 (No. 2), 181-342 (July 1993).

[20] Lateral offsets of the phase plate $-2.5,-1.67,-1,0,1$, $1.67,2.5$ in $\mu \mathrm{m}$.

[21] J. R. Fienup, Appl. Opt. 36, 8352 (1997).

[22] See EPAPS Document No. E-PRLTAO-100-056815 for computer simulations of the reconstruction. For more information on EPAPS, see http://www.aip.org/pubservs/ epaps.html. 